\title{
Analytic Formulation of Transmission Light Intensity of Hole Blockers in Intensity-based Polymer Optical Fiber Sensors
}

\author{
Il-Bum Kwon', Chi-Yeop Kim¹, Chan-Wook Shim², Dusun Hwang² and Yungjoo Chung²,+
}

\begin{abstract}
Intensity-based optical fiber sensors are devised using a blocker which is located between two polymer optical fibers(POFs), one fiber is light-in and the other is light-out. This blocker is moved by an external displacement. Therefore, finding a general formulation of the relation between this displacement and transmission light intensity of various blockers is important to help develop intensity-based optical fiber sensors. In this paper, we consider blockers with arbitrary shapes from circular holes to inclined angled blockers. The transmission light intensities of such blockers should be determined by this generalized equation. In order to verify this equation, the calculated intensities of the blockers are compared with the values acquired from experiment. In the comparison, it is shown that the analytic equation can give the exact values of the transmitted light intensities for the assorted blockers. The range of the displacement measurement is also shown to be about 6 times of the radius of the hole in the case of a 9 degree inclined angle blocker.
\end{abstract}

Keywords : Intensity-Based Optical Fiber Sensor, POF, Blocker, Displacement

\section{INTRODUCTION}

Strain sensors have been widely used in structural health monitoring(SHM) for measuring stress levels in an early stage of structural failure. Compared with conventional electrical strain gages, fiber optic strain sensors have many advantages, such as electro-magnetic immunity and resistance to chemical corrosion. Therefore, in the past few years, there has been intense works on the development of fiber optic strain sensors[1-4]. These most of the developed sensors are very sensitive in measuring strain. However, these sensors are not widely used because they are very expensive. Intensity based fiber optic sensors using POF(Polymer Optical Fiber) can offer a solution to this price problem $[5,6]$. These sensors need a blocker which keeps the light off due to an external perturbation like a displacement, which can be transformed into a strain value by dividing that value by a reference length. When intensity based sensors have light blockers, the transmission light intensity should be studied according to an external measurable parameter, like displacement.

${ }^{1}$ Center for Safety Measurement, Korea Research Institute of Standards and Science, Yuseong-gu, Daejeon, 305-340, South Korea

${ }^{2}$ Department of Information and Communication Engineering, Gwangju Institute of Science and Technology, Buk-gu, Gwangju, , South Korea +Corresponding author: ychung@gist.ac.kr

(Received : Feb. 15. 2011, Revised : May. 17. 2011, Accepted : Jun. 18. 2011)
Therefore, in this paper, an analytic formulation is proposed to determine the transmission light intensity of certain light blockers in the light path, and these calculated results are compared with the experimental results of POF sensors with various blockers, which are of either a circular hole or an inclined angle type.

\section{FORMULATION OF TRANSMISSION LIGHT INTENSITY OF HOLE BLOCKERS}

POF sensors are composed of light-in polymer optical fiber and light-out polymer optical fiber, and one blocker, which cuts off the light through two fibers, as shown in Fig. 1. When the light travels through the blocker between those two fibers, the displacement of the blocker changes the transmission light intensity. As such, it is important to formulate an equation where the transmission light intensity is related to the displacement of the blocker in order to implement intensity-based sensors effectively.

The light energy distribution on the cross section of an optical fiber has been studied by many researchers[7-10]. According to this research, polymer optical fibers with a core diameter over $1 \mathrm{~mm}$, usually have a uniform intensity distribution[10]. This result leads us to conlcude that the cross sectional area of a POF is equal with the intensity of that POF. That is, the light energy distribution is 
homogenous over the cross sectional area of the POF. We can calculate the transmission light intensity by determining the area of the light beam passing through the blocker. Considering convenience in manufacturing the blockers, it would be better to choose a simply shaped blocker such as a hole or an inclined flat plate as shown in Fig. 1. For formulation purposes we consider the edge of the flat plate to be a hole with infinite diameter. When the blocker is caused to move in a horizontal direction by an external parameter, such as displacement, the transmission light intensity is changed by the change of the intersection area between the lead-in POF and lead-out POF.

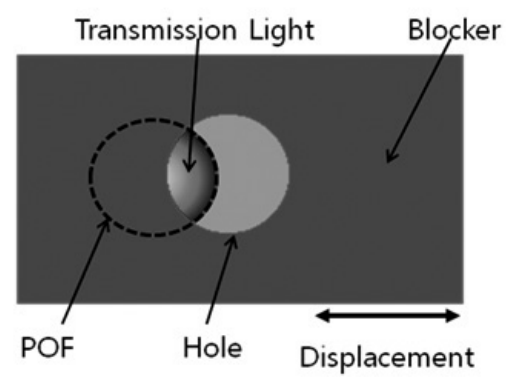

(a) Hole blocker

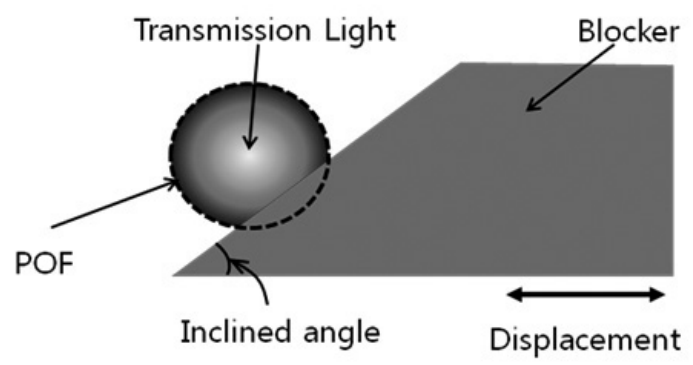

(b) Inclined angle blocker

Fig. 1. Light blockers between lead-in POF and lead-out POF.

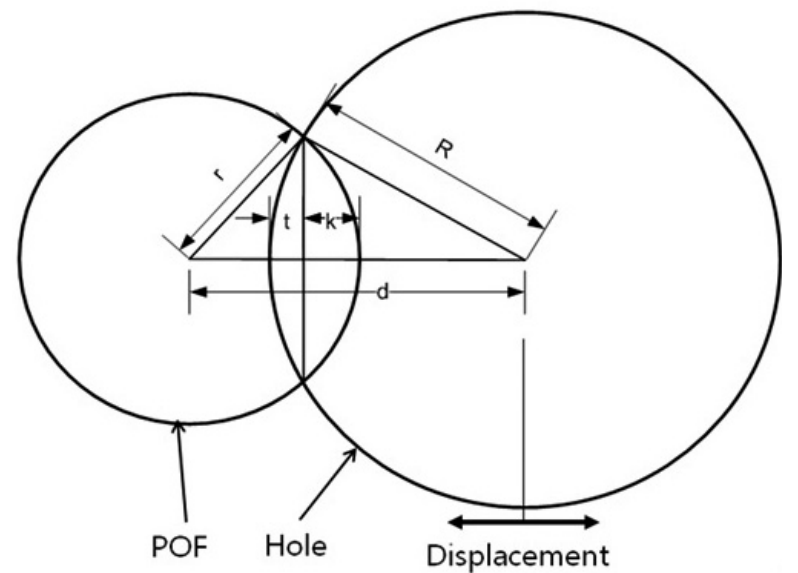

Fig. 2. Schematic drawing of POF and the hole in the hole blocker.
Therefore, the sensor output i.e., the transmission light intensity, can be determined by the analysis of the intersection area. At first, the transmission light intensity of the hole blocker can be derived from the integration of the cross sectional area of the intersection between the two circles in Fig. 2. If the shape of the blocker has a hole of radius $R$, the resulting light transmission intensity is the same as the output signal of the POF sensor. As such, this output can be obtained according to the blocker hole radius, $\mathrm{R}$. The overlapping intersection and the circular cross section are shown in Fig. 2. The smaller circle of radius $r$ is the cross section of the POF and the other circle, of radius $\mathrm{R}$, is the hole of the blocker. The overlap area of the two cross sections can be expressed as a function of $\mathrm{d}$ which is the distance between the center of the POF cross section and the center of the blocker's hole. This area has a direct relation to the light transmission intensity between the two POFs after passing through the blocker. Before calculating the intersection area of the two cross sections, $t$ and $\mathrm{k}$ in terms of $\mathrm{R}, \mathrm{r}$ and $\mathrm{d}$ is given by

$$
\begin{gathered}
t=R-\sqrt{R^{2}-h^{2}}, k=r-\sqrt{r^{2}-h^{2}}, r+R=d+t+k \\
t=\frac{r^{2}-(d-R)^{2}}{2 d}, k=\frac{-(d-r)^{2}+R^{2}}{2 d}
\end{gathered}
$$

The area $A_{1}$ in the POF cross section of radius $r$ is given by

$$
\begin{aligned}
& A_{1}=2 \int_{r-k}^{r} \sqrt{r^{2}-x^{2}} d x \\
& =\frac{1}{2}\left(2 \sqrt{k(2 r-k)}(k-r)+\pi r^{2}-2 r^{2} \tan ^{-1}\left[\frac{-k+r}{\sqrt{k(2 r-k)}}\right]\right)
\end{aligned}
$$

Also, the area $A_{2}$ in the blocker's hole of radius $R$ is given by

$$
\begin{aligned}
& A_{2}=2 \int_{R-t}^{R} \sqrt{R^{2}-x^{2}} d x \\
& =\frac{1}{2}\left(\pi R^{2}+2 \sqrt{(2 R-t) t}(-R+t)-2 R^{2} \tan ^{-1}\left[\frac{R-t}{\sqrt{(2 R-t) t}}\right]\right)
\end{aligned}
$$

The total intersection area between the POF cross section and the blocker's hole is expressed by the sum of $A_{1}$ and $\mathrm{A}_{2}$. Then, the normalized transmission light intensity, In, can be expresses using total intersection area $A_{1}+A_{2}$, and the area $\mathrm{A}_{0}$ of the POF cross section. This gives us 


$$
\begin{aligned}
& I_{n}=\frac{I}{I_{0}}=\frac{A_{1}+A_{2}}{A_{0}} \\
& =\frac{1}{2}\left(\frac{r^{2}+R^{2}}{r^{2}}\right)-\frac{1}{2 \pi r^{2}} \sqrt{d^{2}-(-r+R)^{2}\left(-d^{2}+(r+R)^{2}\right)} \\
& -\frac{1}{\pi} \tan ^{-1}\left[\frac{d^{2}+r^{2}-R^{2}}{\sqrt{d^{2}-(-r+R)^{2}\left(-d^{2}+(r+R)^{2}\right)}}\right]-\frac{R^{2}}{\pi r^{2}} \tan ^{-1}\left[\frac{d^{2}-r^{2}+R^{2}}{\sqrt{d^{2}-(-r+R)^{2}\left(-d^{2}+(r+R)^{2}\right)}}\right.
\end{aligned}
$$

where the distance range of $\mathrm{d}$ is $R-r \leq d \leq R+r$. The distance of $\mathrm{d}$ is defined as the distance between the center positions of the two cross sections. When the hole blocker is considered, the displacement, $\delta$, of the blocker is same as the distance change, $\Delta \mathrm{d}$. However, if there is a need to calculate the case of an inclined angle blocker as shown in Fig. 1 (b), then it should be considered that the blocker has a hole of very big radius $R$, and the direction of displacement, $\delta$, of the inclined angle blocker has some amount of inclined angle, $\theta$ from the direction of the distance change, $\Delta \mathrm{d}$, shown in equation (5).

$$
\delta=\frac{\Delta d}{\sin \theta}
$$

When the inclined angle is 90 degree, the displacement is same as the distance change.

The normalized transmission light intensities of the hole blockers are calculated with the various hole radius ratio, $\mathrm{R} / \mathrm{r}=1,2, \ldots, 10$, of the blockers shown in Fig 4 . In case of $\mathrm{R} / \mathrm{r}=1$, the normalized transmission light intensity has the maximum at $\delta / \mathrm{r}=1$. When the displacement is same as the radius of the POF and $\mathrm{R} / \mathrm{r}=1$, the normoalized transmission light intensity is linearly changed by the displacement. This means, in this case, that the sensor keeps a linear relationship although the cross section of the POF mostly meets the cross section of the blocker's hole. However, when the radius ratio is $2,3, \sim 10$, the linearity is not maintained at $\delta / \mathrm{r}=1$.

If the radius of $R$ is much larger than the radius of $r$ in equation (4), the shape of the blocker can be considered as vertical. If the direction of the distance also makes some angle with the displacement of the blocker, then the equation (4) gives the transmission light intensity by an inclined angle blocker, as shown in Fig. 1 (b).

\section{EXPERIMENT}

A basic experiment was also conducted to find the variation of transmission light intensity according to various blockers with holes or inclined angles and compare the results with the theoretical results. The experimental setup shown in Fig. 3 was used. The POF used in this research is based on a 980 um core diameter multimode and step index fiber(GH 4001) supplied by Mitsubishi Rayon Co., LTD. The light source is a red $\operatorname{LED}(650 \mathrm{~nm})$ and the receiver of the transmitted light with optical loss is the model PDA 36A(ThorLAB) receiver, which is an amplified, switchable-gain, silicon detector designed for the detection of light signals over a $350 \mathrm{~nm}-1100 \mathrm{~nm}$ wavelength range. The variation in intensity of the transmitted light is measured by a photo receiver. These intensity variations are related to the movement of a blocker over a distance interval of about $0.3 \mathrm{~mm}$.

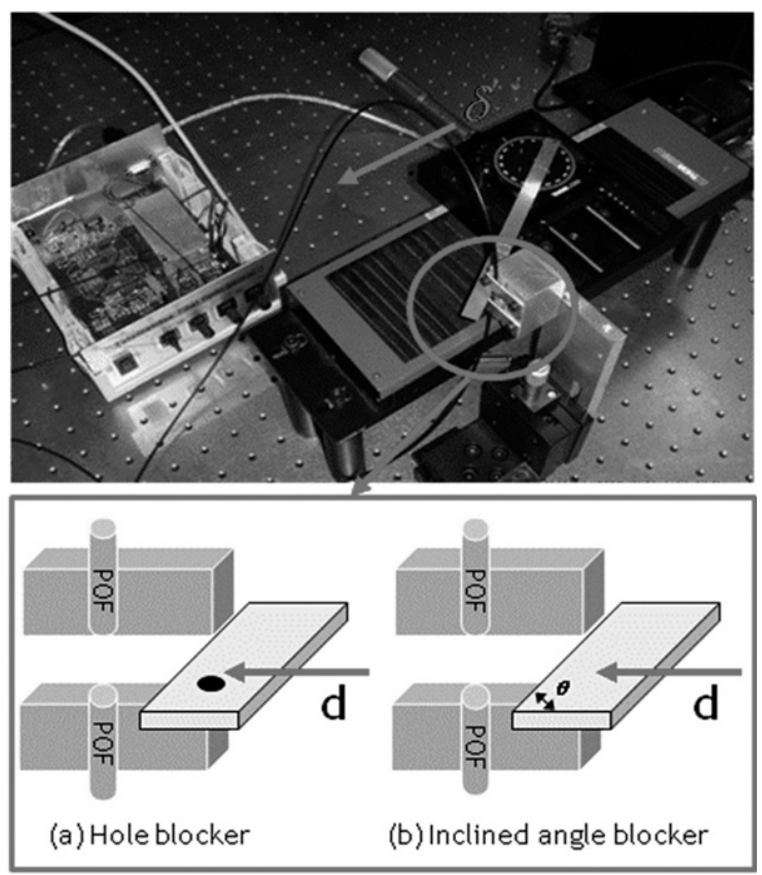

Fig. 3. Experimental setup of an intensity-based POF sensor with (a) a hole blocker and (b) an inclined angle blocker.

\section{DISCUSSION}

The experimental result of the $\mathrm{R} / \mathrm{r}=1.15$ hole blocker are shown as squared dots in Fig. 4 and is compared to the analytical results. In this comparison, the line of the analytic result is well correlated with the dots of the experimental result. Therefore, the analytic equation (4) can be used to determine the transmission light intensity while using a hole type blocker in intensity-based sensors. Meanwhile, Fig. 5 shows the analytical and experimental results which prove that the modulation of light intensity is 
due to the blocking, at the point between two POFs, having an inclination of various degrees. The analytic results for the inclined angle blockers are obtained from equation (4) using a very large radius, R. Looking at these results, we can see a linear relationship between the transmission light intensity and the displacement is found in the central area. The intensity change of a 60 degree blocker is similar to that of a 90 degree vertical blocker. If the displacement sensor needs to have high sensitivity, the angle of the inclined blocker should be limited to less than 60 degrees. On the other hand, if the range of the displacement ratio of the POF displacement sensor is over 1, the angle of the blocker should be larger than 60 degrees. Also, the range of displacement measurements is shown at about 6 times of the radius of the hole in case of a 9 degree inclined angle blocker. In these discussions, equation (4) gave us the transmission light intensity of various hole blockers and also inclined angle blockers.

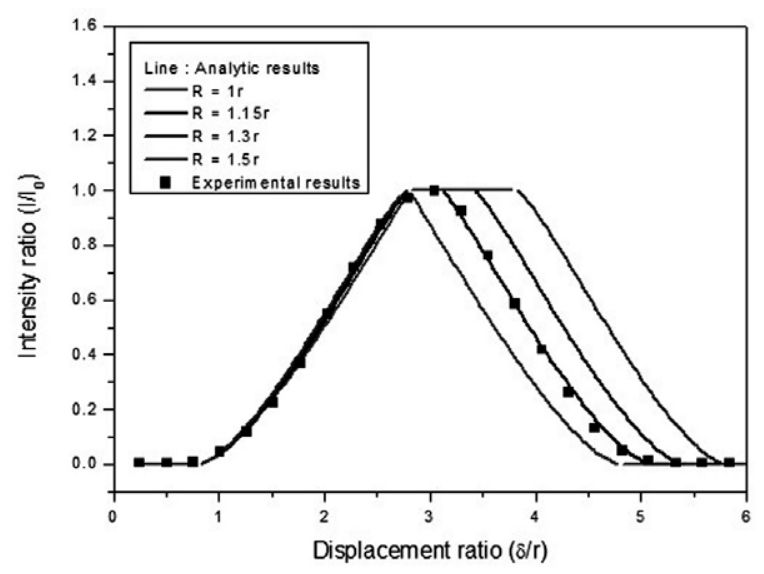

Fig. 4. Transmission light intensity according to the displacement of the hole blocker.

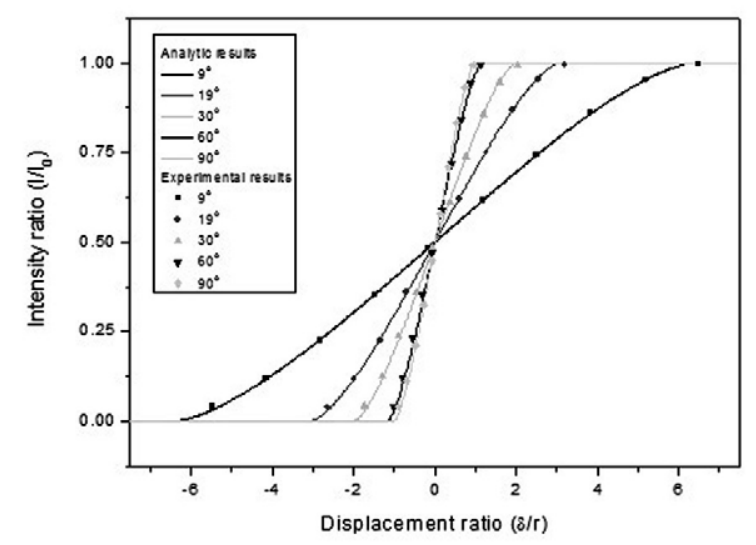

Fig. 5. Transmission light intensity according to the displacement of the inclined angle blocker.

\section{CONCLUSION}

In order to develop blocker-type intensity-based optical fiber sensors, it is necessary to calculate the transmission light intensity. In this paper, a formulation was provided to give a very convenient method of determining the transmission light intensity when using inclined angle and hole blockers. The analytic calculations and experimental results showed that most of the blockers showed some nonlinear parts at the start and the end of the operating range of the displacement except for the hole blocker with a radius ratio, $\mathrm{R} / \mathrm{r}=1$. The sensor response of inclined angle blockers is big in the angle range between 60 and 90 degrees. However, if the displacement ratio is larger than 1 , the angle of the blocker should be less than 60 degrees. The range of displacement measurements was also shown to be about 6 times the radius of the hole in the case of the 9 degree inclined angle blocker. Therefore, this new analytical formulation is expected to give a basic analytical tool to researchers developing blocker-type intensity-based optical fiber sensors.

\section{ACKNOWLEDGEMENT}

This work was supported by Telemetrics Project (1001678) under Ministry of Knowledge and Economics, and KRISS project, "Development of Advanced Measurement Technology for Public Safety."

\section{REFERENCES}

[1] Jong H. Chow, David E. McClelland, and Malcolm B. Gray, "Demonstration of a passive subpicostrain fiber strain sensor," Optics Letters, vol. 30, no. 15, pp. 19231925, 2005.

[2] Graham Duck and Myo M. Ohn, "Distributed Bragg grating sensing with a direct group-delay measurement technique," Optics Letters, vol. 25, no. 2, pp. 90-92, 2000.

[3] P. Moyo, J. M. W. Brownjohn, R. Suresh, and S. C. Tjin, "Development of fiber Bragg grating sensors for monitoring civil infrastructure," Engineering Structures, vol. 27, pp. 1828-1834, 2005.

[4] J. I. Koh, H. J. Bang, C. G. Kim, and C. S. Hong, "Simultaneous measurement of strain and damage signal of composite structures using a fiber Bragg 
grating sensor," Smart Mater. Struct. vol. 14, pp. 658$663,2005$.

[5] D. Kalymnios, "Plastic optical fibres(POF) in sensingcurrent status and prospects," Proceeding of SPIE, vol. 5855, pp. 1-4, 2005.

[6] K. S. C. Kuang, S. T. Quek, and M. Maalej, "Assessment of an extrinsic polymer-based optical fibre sensor for structural health monitoring," Measurement Science and Technology, vol. 15, pp. 2133-2141, 2004.

[7] H. Fujii, T. Asakura, T. Matsumoto, and T. Ohura, "Output power distribution of a large core optical fiber," IEEE J. Lightwave Technol, vol. 38, pp. 1057 1062, 1984.
[8] H. Golnabi, "Precise CCD image and analysis for planar laser induced fluorescence experiments," Optics Laser Technol, vol. 38, pp. 152-161, 2006.

[9] C. P. Tsekrekos, R. W. Smink, B. P. De Hon, A. G. Tijhuis, and A. M. J. Koonen, "Near-field intensity pattern at the output of silica-based graded-index multimode fibers under selective excitation with a single-mode fiber," Optics Express, vol. 15, pp. 36563664, 2007.

[10] A. Asadpour, and H. Golnabi, "Beam profile and image transfer study in multimode optical fiber coupling," Journal of Applied Sciences, vol. 8, pp. 4210-4214, 2008.

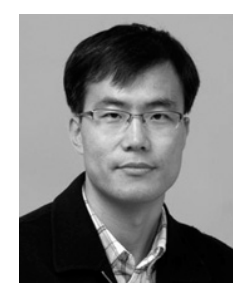

Il-Bum Kwon received his $\mathrm{Ph} . \mathrm{D}$. from Korea Advanced Institute of Science and Technology(KAIST) in 1997. His major field of study was aerospace engineering with specialization in structural health monitoring using fiber optic sensors. He has been employed by Research Institute of Industrial Science and Technology(RIST/POSCO) since February 1989 and conducts research in rolling research laboratory until August 1992. Currently, he is working for Center for Safety Measurement in Korea Research Institute of Standards and Science since March 1997. His research interests include fiber optic sensors for structural health monitoring, optical fiber and optical communications for sensor network.

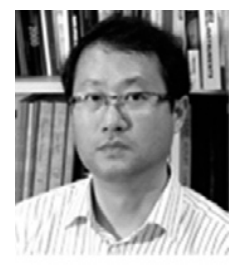

Yungjoo Chung received his M.A. degree from Princeton University at 1985, and his Ph.D degree from Princeton University at 1989. He worked for Argon National Laboratory during 1989 - 1996. He is now a professor of department of information and communication engineering of Gwangju Institute of Science and Technology from 2001. His research includes fiber Bragg gratings, optical fiber devices, optical communication and sensing.
Chi-Yeop Kim received his B.E. degree at department of electronic engineering from Inha University in 1984. He worked for LG electronics from 1984 to 1986 . He is now working for Korea Research Institute of Standards and Science since 1986. His roll is the development of electronic circuit boards for sensors. His interesting research field is wireless sensors.

Chan-Wook Shim received his Master's degree of information and communication engineering from Gwanju Insitute of Science and Technology at 2009. He is now working for Dongbu Hynix Co. in Korea. His research interests are fiber optic sensors and optical instrumentation.

Duseon Hwang received his Master's degree and Ph.D from Gwangju Institute of Science and Technology at 2003, 2010, respectively. He worked on developing several fiber optic sensors during his Ph.D works. He is now working for LG research institute. His researches include fiber optic devices and sensors. 